\title{
Sonographic Diagnosis of Neonatal Diaphragmatic Inversion
}

\author{
Dorit D. Adler MD, Barry I. Samuels, MD, and Caroline E. Blane, MD
}

Respiratory distress in the neonate is a common problem. An inverted hemidiaphragm may be a significant factor contributing to the respiratory distress. ${ }^{1}$ Inversion of both hemidiaphragms has been reported in all age groups. ${ }^{2}$ Three papers in the literature report a total of 15 adults with right hemidiaphragm inversion diagnosed sonographically. ${ }^{3-5}$ This case report is of a neonate with left hemidiaphragmatic inversion diagnosed by ultrasound.

\section{CASE REPORT}

A 3-kg-girl was delivered by emergency cesarean section at 42 weeks of gestation because of heavy meconium staining of the amniotic fluid. The infant was transferred to the Holden neonatal intensive care nursery with the diagnosis of severe birth asphyxia, meconium aspiration, oliguria, and seizures.

High pressure ventilation requirements led to the complications of interstitial pulmonary emphysema, pneumothorax, and pneumomediastinum. Within the first 2 days of life, six chest tubes had been placed for recurrent pneumothoraxes. Because oxygenation remained poor and pulmonary complications were severe, the child was placed on extracorporeal membrane oxygenation (ECMO) at 56 hours of age. This necessitated anticoagulant therapy.

At 5 days of age increasing respiratory distress, bleeding from chest tubes, and deteriorating status prompted the clinicians to order sonography of the abdomen and head. The abdominal and cranial ultrasound examinations were unremarkable. Chest radiographs and sonography

From the Department of Radiology, University of Michigan Medical Center, Ann Arbor, Mich. Manuscript received January 17, 1983; manuscript accepted May 13, 1983. For reprints contact Barry I. Samuels, MD, Department of Radiology, Box 013, University of Michigan Medical Center, Ann Arbor, Mich. 48109.
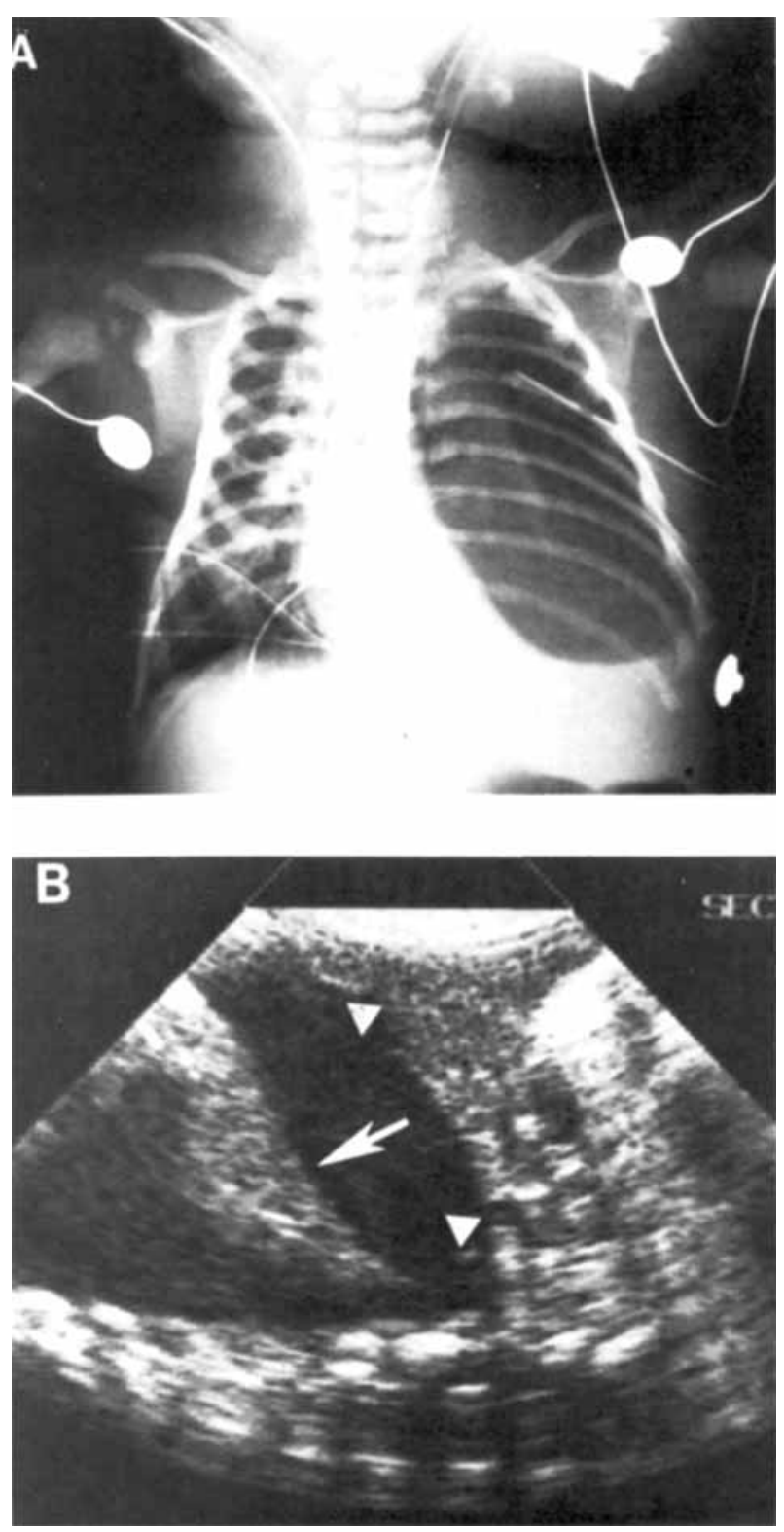

FIGURE 1. (A) Portable supine radiograph revealed complete collapse of the left lung with an associated large left pleural effusion. Note the left hemidiaphragm is not identified. (B) Real-time longitudinal chest sonogram demonstrating left pulmonary collapse (arrow), massive pleural fluid, and an inverted left hemidiaphragm (arrowheads). 
showed collapse of the left lung and a large left pleural effusion suggesting a hemopneumothorax. An inverted left hemidiaphragm was also identified with paradoxical motion [Fig. 1(A, B)]. The latter was believed to be contributing to the respiratory distress. The child was taken to the operating room immediately and left thoracotomy performed to achieve hemostasis. The hemopneumothorax resolved and the respiratory distress improved.

\section{DISCUSSION}

Diaphragmatic inversion can be a significant factor in the production of respiratory distress. ${ }^{1}$ The association between flattening and inversion of the diaphragm and the severity of dyspnea has been reported. ${ }^{6}$ Diaphragmatic inversion is usually associated with pleural effusion. ${ }^{6}$ This combination makes evaluation of the chest radiograph difficult. The contour of the inverted hemidiaphragm is usually obscured by the overlying pleural effusion.

Sonographic evaluation of other diaphragmatic abnormalities in infants has been reported. ${ }^{7}$ Ultrasound can provide information on the diaphragmatic position and contour and real- time sonography can demonstrate diaphragmatic motion. Sonographic diagnosis of inversion of a hemidiaphragm with paradoxical motion provides additional information for evaluation and treatment of the neonate with respiratory distress.

\section{REFERENCES}

1. Rogers CI, Meredith HC: Osler revisited: An unusual cause of inversion of the diaphragm. Radiology 125:596, December 1977.

2. Felson B: Chest Roentgenology. Philadelphia; Saunders, 1973.

3. Bouvier M, Frech M, Vivier G, et al: Inversion diaphragmatique droit lors des epanchements pleuraux abondants. J Radiol 60:739-742, 1979.

4. Subramanyam BR, Raghavendra BN, Lefleur RS: Sonography of the inverted right hemidiaphragm. Am J Roentgenol 136:1004-1006, 1981.

5. Lowe SH, Cosgrove DO, Joseph AEA: Inversion of the right hemidiaphragm shown on ultrasound examination. Br J Radiol 54:754-757, 1981.

6. Mulvey RB: The effect of pleural fluid on the diaphragm. Radiology 84:1080-1085, 1965.

7. Merten DF, Bowie JD, Kirks DR, et al: Anteromedial diaphragmatic defects in infancy: Current approaches to diagnostic imaging. Pediatr Radiol 142:361-365, 1982. 